\title{
Network meta-analysis of responses to DPP-4 and SGLT-2 inhibitors: What next after metformin with a baseline HbA1c $\leq 8 \%$ ?
}

\section{David W. Strain ${ }^{1}$, Merlin C. Thomas ${ }^{2}$, James E. Foley ${ }^{3}$, Päivi M. Paldánius ${ }^{4}$}

'University of Exeter Medical School, Exeter, UK; ${ }^{2 B}$ aker IDI Heart and Diabetes Institute, Melbourne, Australia; ${ }^{3}$ Novartis Pharmaceuticals Corporation, East Hanover, NJ, USA; ${ }^{4}$ Novartis Pharma AG, Basel, Switzerland

\section{CONCLUSIONS}

- For delivery of individualised care, treatment choices should consider whether we are aiming to reduce cardiovascular risk in people with established cardiovascular disease or reducing microvascular risk in early diabetes by targeting hyperglycaemia.

- Results of this analysis are consistent with the other studies in which glucose lowering with DPP-4 inhibitors was greater than with SGLT-2 inhibitors in patients with $\mathrm{HbAlc}<8 \%$, although no individual patient data from these studies was included in the present analysis.

- With broadly similar tolerability, in those with early disease, existing data would recommend a choice between DPP-4 inhibitors and SGLT-2 inhibitors in people that we would not wish to risk hypoglycaemia or further weight gain.

- The available literature suggests that, in patients with baseline HbAlc $\leq 8 \%$, DPP-4 inhibitors may be more potent than SGLT-2 inhibitors at lowering glucose.

\section{INTRODUCTION}

- Guidelines recommend early and aggressive treatment in younger patients with shorter duration of type 2 diabetes mellitus (T2DM) after metformin failure to prevent secondary complications.

- Dipeptidyl peptidase-4 (DPP-4) inhibitors ${ }^{2,3}$ and sodium-glucose co-transporter 2 (SGLT-2) inhibitors ${ }^{4}$ demonstrate baseline-dependent reduction in glycated haemoglobin $(\mathrm{HbA1c})$ in patients with T2DM, albeit with different mechanisms of action.

- In the majority of people with early diabetes, however, the choice between SGLT-2 inhibitor and DPP-4 inhibitor is driven by their ability to reduce blood glucose levels.

- As SGLT-2 inhibitors are dependent on glycosuria from circulating glucose ${ }^{4}$ their dominance over DPP-4 inhibitors at high baseline $\mathrm{HbAlc}$ is unchallenged. However, where clinical inertia does not exist and therapy is being escalated at an appropriate time ( $\mathrm{HbAlc} \leq 8 \%)$, the comparative effectiveness of these agents remains unclear.

\section{OBJECTIVE}

- To explore the relative benefits of DPP-4 inhibitors and SGLT-2 inhibitors in patients with T2DM, inadequately controlled with monotherapy at $\mathrm{HbA1c}$ levels indicated for therapy escalation by most guidelines.

\section{METHODS}

- A network meta-analysis based on a systematic literature review was conducted to identify studies comparing DPP-4 inhibitors and SGLT-2 inhibitors in patients with T2DM, as add-on to metformin or to metformin and sulphonylurea, exploring the $\mathrm{HbAlc}$ reduction from a baseline $\leq 8 \%$.

- The review protocol was prepared based on the PRISMA good reporting guidelines for systematic review and meta-analysis protocols. ${ }^{5}$

- The patient population, intervention, comparators, outcomes, and study design (PICOS) for this review are presented in Figure 1.

- A base-case analysis of 12-26 weeks and a sensitivity analysis of 12-52 weeks (inclusive) were performed using a fixed and random effects model. The mean differences in change from baseline between intervention and control groups were used as model inputs.

\section{Figure 1. PICOS elements}

\section{Adult patients with T2DM inadequately controlled (HbA1c $\leq 8 \%$ ) on Patients stable metformin monotherapy and diet + exercise program}

\section{Others:}

Primary intervention: Vildagliptin

DPP-4 inhibitors: alogliptin, linagliptin, saxagliptin, sitagliptin

Interventions teneligliptin

- SGLT-2 inhibitors: canagliflozin, dapagliflozin, empagliflozin - All interventions in conjunction with metformin

\section{Metformin plus:}

- Placebo

- No intervention

Sulphonylureas (e.g. glimepiride, glipizide, meglitinides)

Comparators - Any of the above SGLT-2 inhibitor/DPP-4 inhibitor interventions

\section{- Change in HbAlc from baseline to study end \\ - Weight change from baseline to study end \\ - Change in FPG from baseline to study end}

Study design Phase III and IV randomised controlled trials

DPP-4, dipeptidyl peptidase-4; FPG, fasting plasma glucose; HbAlc, glycated haemoglobin; PICOS, population, intervention, comparators, outcomes, and study design; SGLT-2, sodium-glucose co-transporter 2; T2DM, type 2 diabetes mellitus

\section{RESULTS}

- Outcome data on patients with baseline HbAlc $\leq 8 \%$ was found in 19 phase III/IV randomised controlled trials (Figure 2), 13 on patients receiving only metformin as background therapy and 5 on patients receiving metformin + sulphonylurea as background therapy.

Figure 2. PRISMA flow chart demonstrating literature search and study selection

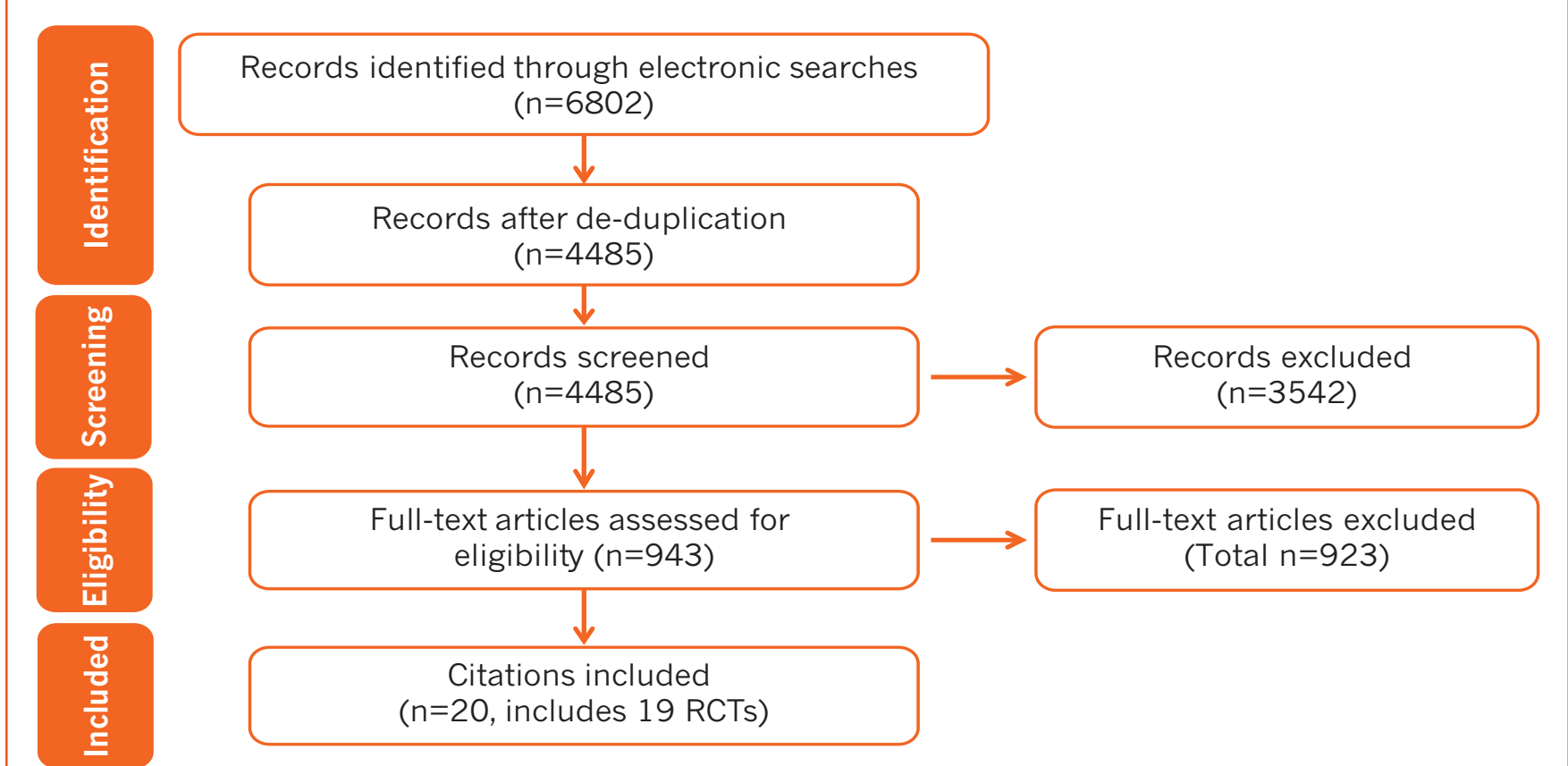

PRISMA, preferred reporting items of systematic reviews and meta-analyses; RCT, randomised controlled trials

- Among DPP-4 inhibitors, we only had access to patient level data from vildagliptin, therefore only this was utilised.

- For the background metformin monotherapy network, the fixed effects model indicated that vildagliptin $100 \mathrm{mg} /$ day and SGLT-2 inhibitors were more effective than placebo and (escalation of) metformin.

- For the background metformin and sulphonylurea network, the fixed effects model indicated that vildagliptin $100 \mathrm{mg}$ is more effective than placebo, dapagliflozin 10 mg, canagliflozin $100 \mathrm{mg}$ and comparable to canagliflozin 300 mg (Figure 3).

- We had no access to patient level data for reports comparing efficacy of DPP-4 inhibitors and empagliflozin (10 or $25 \mathrm{mg}$ ).

- No manuscripts reported differences in weight at these baseline HbAlcs.

Figure 3. Base-case analysis (patients receiving metformin and sulphonylurea as background therapy, fixed effect model). Forest plot versus vildagliptin $100 \mathrm{mg} /$ day

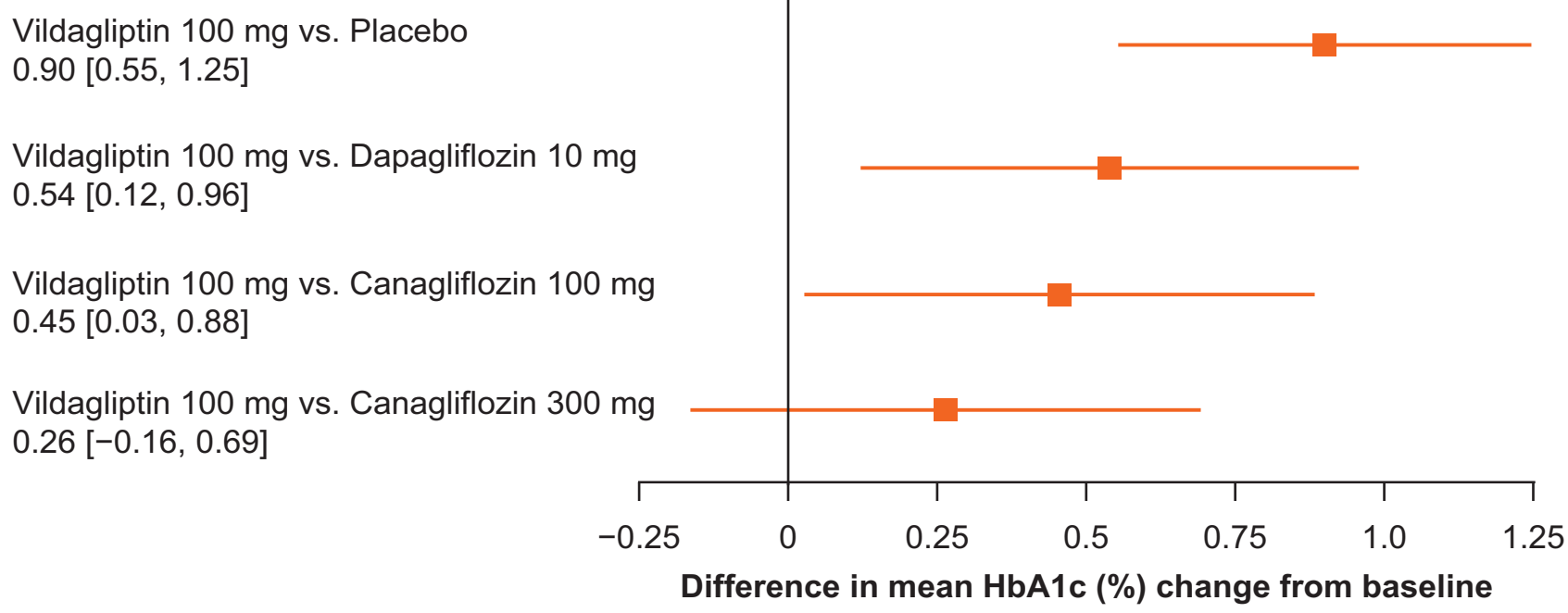

Values are mean differences in change from baseline in $\mathrm{HbAlc}(\%)$ and $95 \%$ credible intervals

$\mathrm{HbAlc}$, glycated haemoglobin

\section{References}

1. Inzucchi SE, et al. Diabetes Care. 2015;38:140-9.

4. Abdul-Ghani MA, et al. Endocr Rev. 2011;32:515-31. 2. Eckerle Mize DL and Salehi M. Curr Diab Rep. 2013;13:307-18. 5. Moher D, et al. Syst Rev. 2015;4:1.

3. Röhrborn D, et al. Front Immunol. 2015;6:386.

Disclosures

The study was funded by Novartis. WDS received grants and personal fees from Novartis during the conduct of this study, personal fees from Boehringer Ingelheim, Pfizer, and personal fees from Novo-Nordisk, outside the submitted work. MCT received honoraria for educational symposia

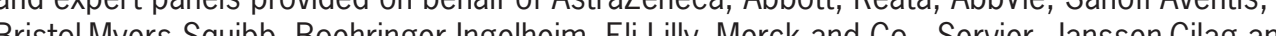

\section{Acknowledgements}

The authors thank Sashi Kiran Goteti and Vinod Kumar Goshamahal, Novartis Healthcare Pvt. Ltd, Hyderabad, India, for medical writing support and graphic design support, respectively. 